\title{
Dynamic calibration of higher eigenmode parameters of a cantilever in atomic force microscopy by using tip-surface interactions
}

\author{
Stanislav S. Borysov ${ }^{\star 1}, 2,3$, Daniel Forchheimer ${ }^{1}$ and David B. Haviland ${ }^{1}$
}

\section{Full Research Paper}

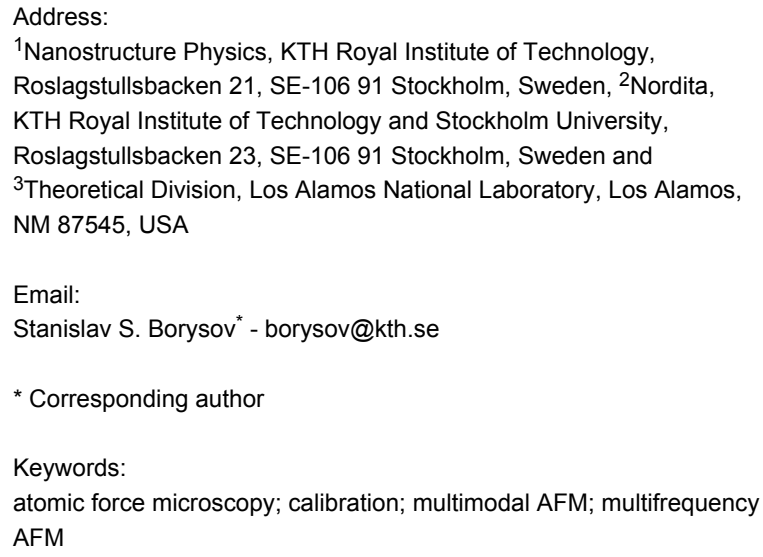

Beilstein J. Nanotechnol. 2014, 5, 1899-1904. doi:10.3762/bjnano.5.200

Received: 10 June 2014

Accepted: 01 October 2014

Published: 29 October 2014

This manuscript is part of the Thematic Series "Advanced atomic force microscopy techniques II".

Guest Editors: T. Glatzel and T. Schimmel

(C) 2014 Borysov et al; licensee Beilstein-Institut.

License and terms: see end of document.

\begin{abstract}
We present a theoretical framework for the dynamic calibration of the higher eigenmode parameters (stiffness and optical lever inverse responsivity) of a cantilever. The method is based on the tip-surface force reconstruction technique and does not require any prior knowledge of the eigenmode shape or the particular form of the tip-surface interaction. The calibration method proposed requires a single-point force measurement by using a multimodal drive and its accuracy is independent of the unknown physical amplitude of a higher eigenmode.
\end{abstract}

\section{Introduction}

Atomic force microscopy [1] (AFM) is one of the primary methods of surface analysis with resolution at the nanometer scale. In a conventional AFM an object is scanned by using a microcantilever with a sharp tip at the free end. Measuring cantilever deflections allows not only for the reconstruction of the surface topography but also provides insight into various material properties [2,3]. If deflection is measured near one of the cantilevers resonance frequencies, an enhanced force sensi- tivity is achieved due to multiplication by the sharply peaked cantilever transfer function. Measurement of response at multiple eigenmodes can provide additional information about the tip-surface interactions [4-11].

The optical detection system [12] common to most AFM systems leverages a laser beam reflected from the cantilever, measuring the slope rather than its vertical deflection. This 
underlying principle leads to the measured voltage at the detector being dependent on the geometric shape of the excited eigenmode (Figure 1). While determination of the stiffness and optical lever inverse resposivity (inverse magnitude of the response function of the optical lever [m/V], also known as "inverse optical lever sensitivity") of the first flexural eigenmode can be performed with high accuracy using a few welldeveloped techniques [13-21], calibration of the higher eigenmode parameters is still a challenging task. The main problem with the existing theoretical approaches based on the calculation of eigenmode shapes $[18,22]$ is that real cantilevers differ form the underlying solid body mechanical models due to the tip mass $[23,24]$, fabrication inhomogeneities and defects $[25,26]$. In this paper, we propose a method which overcomes these deficiencies.
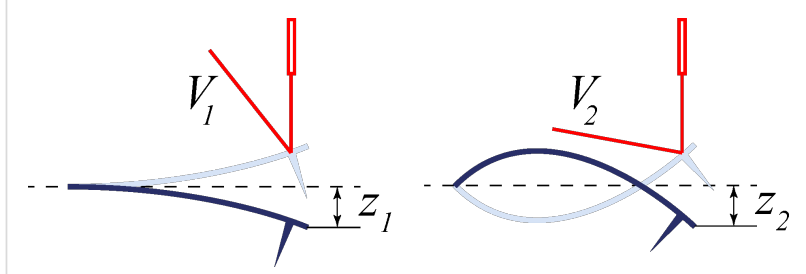

Figure 1: Schematic illustration of the two first flexural eigenmode shapes of a rectangular cantilever and an optical detection system. Measuring of the slope at the free end leads to the situation when the equal vertical tip deflections, $z_{1}=z_{2}$, result in the different detected voltages, $v_{1} \neq V_{2}$. In the case of small deflections, $z_{n}$ is proportional to $V_{n}$ with some coefficient $\alpha_{n}$ called optical lever inverse responsivity.

The method uses the fact that the tip-surface force is equally applied to all eigenmodes. This approximation is suitable unless the characteristic spatial wave length of an eigenmode shape is significantly bigger than the tip-cantilever contact area. Any other force acting on the whole cantilever, e.g., of thermal or electromagnetic nature, should be convoluted with the eigenmode shape, leading to a different definition of the effective dynamic stiffness. Thus, knowledge of the geometry of cantilever is not required to reconstruct the tip-surface force. The framework proposed harnesses a force reconstruction technique inspired by the Intermodulation AFM [27] (ImAFM), which was recently generalized to the multimodal case [28]. It is worth noting that the proposed calibration method is similar to that described in [29], in which stiffness of the second eigenmode is experimentally defined by using consecutive measurements of the frequency shift caused by the tip-surface interaction for different eigenmodes. In contrast, we propose a simultaneous one-point measurement by using a multimodal drive that avoids issues related to the thermal drift [30] and exploits nonlinearities for higher calibration precision.

\section{Results and Discussion Cantilever model}

We consider a point-mass approximation of a cantilever derived from the eigenmode decomposition of its continuum mechanical model, e.g., the Euler-Bernoulli beam theory. Such a reduced system of coupled harmonic oscillators in the Fourier domain has the following form

$$
k_{n} \alpha_{n} \hat{V}_{n}(\omega)=\hat{G}_{n}(\omega)\left[\hat{F}(\omega)+\hat{\mathrm{f}}_{n}(\omega)\right]
$$

where the caret denotes the Fourier transform, $\omega$ is the frequency, $k_{n}$ is the effective dynamic stiffness of the $n$th eigenmode $(n=1, \ldots, N), \alpha_{n}$ is the optical lever inverse responsivity, $V_{n}$ is the measured voltage (corresponding to the eigencoordinate $z_{n}=\alpha_{n} V_{n}$, where total tip deflection is $\left.z=\sum_{n=1}^{N} z_{n}\right)$,

$$
\hat{G}_{n}=\left[1+\left(i / Q_{n}\right)\left(\omega / \omega_{n}\right)-\left(\omega / \omega_{n}\right)^{2}\right]^{-1}
$$

is the linear transfer function of a harmonic oscillator with the resonant frequency $\omega_{n}$ and quality factor $Q_{n}, F$ is a nonlinear tip-surface force and $\mathrm{f}_{n}$ is a drive force. The stiffness is deliberately excluded from the expression for the $G_{n}$ since the parameters $Q_{n}$ and $\omega_{n}$ can be found by employing the thermal calibration method $[14,17]$. Note that if the force amplitudes on the right hand side of Equation 1 are known, one immediately gets $k_{n}$ and $\alpha_{n}$ by taking the absolute values in combination with the equipartition theorem

$$
k_{n}\left\langle z_{n}^{2}\right\rangle=k_{n} \alpha_{n}^{2}\left\langle V_{n}^{2}\right\rangle=k_{\mathrm{B}} T
$$

where $\langle\cdot\rangle$ is a statistical average, $k_{\mathrm{B}}$ is the Boltzmann constant and $T$ is an equilibrium temperature.

\section{Spectral fitting method}

The task at hand requires reconstruction of the forces on the right hand side of Equation 1 from the measured motion spectrum. Firstly, it is possible to remove the unknown drive contribution, $\hat{\mathrm{f}}_{n}$, for each $n$, by means of subtraction of the free oscillations spectrum, $\hat{V}_{n}^{\mathrm{f}}$ (far from the surface, where $F \equiv 0$ ), from the spectrum of the engaged tip motion, $\hat{V}_{n}^{\mathrm{e}}$ (near the surface). It gives the following relationships

$$
k_{n} \alpha_{n} \Delta \hat{V}_{n}=\hat{G}_{n} \hat{F}
$$

where $\Delta \hat{V}_{n} \equiv \hat{V}_{n}^{\mathrm{e}}-\hat{V}_{n}^{\mathrm{f}}$. For the high- $Q$ cantilevers, the measured response near each resonance $\hat{V}_{n}$ may be separately detected with the high signal-to-noise ratio (SNR). Neglecting possible 
surface memory effects, $F$ depends on the tip position $z$ and its velocity $\dot{z}$ only. With this assumption, the force model to be reconstructed has some generic form

$$
\begin{aligned}
\widetilde{F}(z, \dot{z}) & =\sum_{i=0}^{P_{z}} \sum_{j=0}^{P_{z}} g_{i j} z^{i} \dot{z}^{j} \\
& =\sum_{i=0}^{P_{z}} \sum_{j=0}^{P_{z}} g_{i j}\left(\sum_{n=1}^{N} \alpha_{n} V_{n}^{\mathrm{e}}\right)^{i}\left(\sum_{n=1}^{N} \alpha_{n} \dot{V}_{n}^{\mathrm{e}}\right)^{j},
\end{aligned}
$$

with $P=P_{z} P_{z}-1$ unknown parameters $g_{i j}\left(g_{00}\right.$ is excluded because it corresponds to the static force) which can be found by using the spectral fitting method $[31,32]$ : Substitution of Equation 5 in Equation 4 yields a system of linear equations for $g_{i j}$. However, this system becomes nonlinear with respect to the unknown $k_{n}$ and $\alpha_{n}$.

\section{Intermodulation AFM}

Assuming that $\alpha_{1}$ and $k_{1}$ are calibrated by using one of the methods mentioned in the Introduction, the resulting system contains $2(N-1)+P$ unknown variables. Use of the equipartition theorem (Equation 3) for each eigenmode gives us $N-1$ equations and the remaining equations should be defined by using Equation 4 for the known response components in the motion spectrum. If the force acting on a tip over its motion domain is approximately linear $(P=1)$, one drive tone at each resonant frequency is enough to determine the system. However, when the force behaves in a nonlinear way $(P>1)$, as is usually the case, more measurable response components in the frequency domain are needed. The core idea of ImAFM relies on the ability of a nonlinear force to create intermodulation of discrete drive tones in a frequency comb. Driving an eigenmode subject to a nonlinear force on at least two frequencies $\omega_{n 1}^{\mathrm{d}}$ and $\omega_{n 2}^{\mathrm{d}}$ gives a response in the frequency domain not only at these drive frequencies and their higher harmonics but also at their linear combinations $n \omega_{1}^{\mathrm{d}}+m \omega_{2}^{\mathrm{d}}$ ( $n$ and $m$ are integers) called intermodulation products (IMPs). Use of the small base frequency $\delta \omega=\left|\omega_{n 1}^{\mathrm{d}}-\omega_{n 2}^{\mathrm{d}}\right|$ results in the concentration of IMPs close to the resonance, which opens the possibility for their detection with high SNR. This additional information can be used in Equation 4 for the reconstruction of nonlinear conservative and dissipative forces [28,31-33] with the only restriction that IMPs in the different narrow bands near resonances contain the same information about the unknown force parameters [28].

\section{Calculation details}

In the rest of the paper, we consider a bimodal case implying straightforward generalization for $N>2$ eigenmodes. Equation 1 is integrated by using CVODE [34] for two different sets of cantilever parameters from Table 1. The cantilever is excited by using multifrequency drive (specified below) with frequencies being integer multiples of the base frequency $\delta \omega=2 \pi \cdot 0.1 \mathrm{kHz}$. The tip-surface force $F$ is represented by the vdW-DMT model [35] with the nonlinear damping term being exponentially dependent on the tip position [36]

$$
\begin{aligned}
& F=F^{\mathrm{con}}+F^{\mathrm{dis}}, \\
& F^{\mathrm{con}}(z)= \begin{cases}-\frac{H R}{6(z+h)^{2}}, & z+h \geq a_{0} \\
-\frac{H R}{6 a_{0}^{2}}+\frac{4}{3} E^{*} \sqrt{R\left(a_{0}-(z+h)\right)}, & z+h<a_{0}\end{cases} \\
& F^{\text {dis }}(z, \dot{z})=-\gamma_{1} \dot{z} e^{-(z+h) / \lambda_{z}},
\end{aligned}
$$

where $h$ is a reference height. Its conservative part, $F^{\text {con }}$, has four phenomenological parameters: the intermolecular distance $a_{0}=0.3 \mathrm{~nm}$, the Hamaker constant $H=7.1 \times 10^{-20} \mathrm{~J}$, the effective modulus $E^{*}=1.0 \mathrm{GPa}$ and the tip radius $R=10 \mathrm{~nm}$. The dissipative part, $F^{\text {dis }}$, depends on the damping factor $\gamma_{1}=2.2 \times 10^{-7} \mathrm{~kg} / \mathrm{s}$ and the damping decay length $\lambda_{z}=1.5 \mathrm{~nm}$. The force (Equation 6) and its cross-sections are depicted in Figure 2.

\section{Calibration by using a nonlinear tip-surface force}

In order to find $k_{2}$ and $\alpha_{2}$ from the nonlinear system (Equation 3 and Equation 4), we first solve the linear system for the force parameters $g_{i j}$. It is then convenient to compare only the conservative part of the tip-surface force given its non-monotonic

\begin{tabular}{|c|c|c|c|c|c|c|c|c|}
\hline cantilever & $\omega_{1}\left((2 \pi)^{-1} \mathrm{kHz}\right)$ & $\omega_{2} / \omega_{1}$ & $Q_{1}$ & $Q_{2} / Q_{1}$ & $k_{1}(\mathrm{~N} / \mathrm{m})$ & $k_{2} / k_{1}$ & $\alpha_{2} / \alpha_{1}$ & $E(\mathrm{fJ})$ \\
\hline soft & 82.7 & 6.35 & 220.0 & 2.9 & 5.0 & 40.0 & 2.0 & 1.02 \\
\hline stiff & 300.0 & 6.3 & 400.0 & 3.0 & 40.0 & 50.0 & 2.0 & 0.105 \\
\hline
\end{tabular}
behavior. There are two methods to require equality of the reconstructed forces $\widetilde{F}^{(1)}$ (using the band near the first eigenmode) and $\widetilde{F}^{(2)}$ (near the second eigenmode). The first method is to check the difference between the corresponding parame- 


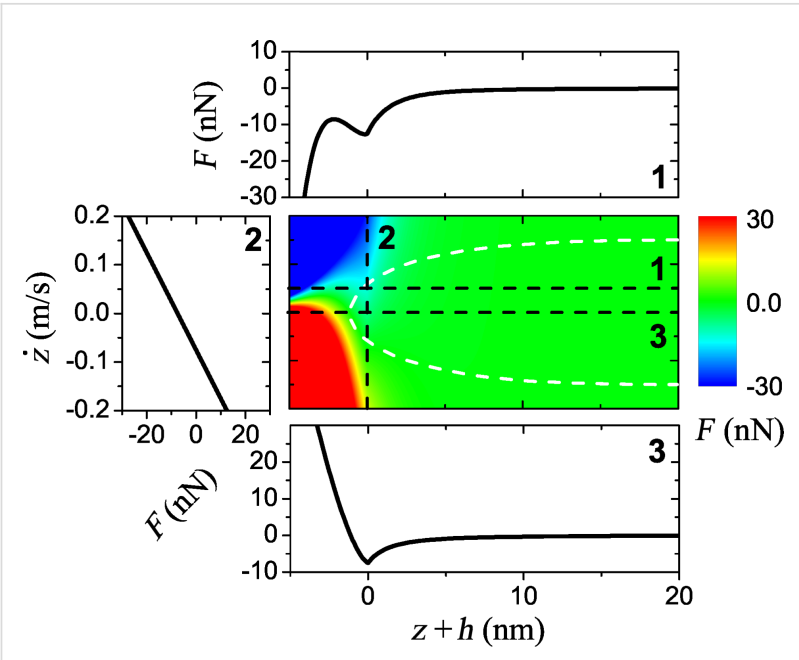

Figure 2: The tip-surface force (Equation 6) used in the simulations. The white dashed line corresponds to a phase space trajectory of the bimodal stiff cantilever with the eigenmode amplitudes $A_{1}=A_{2}=12.5$ $\mathrm{nm}$ and reference height $h=17 \mathrm{~nm}$. Cross-sections for different values of $z$ and $z$ are shown: The projections (1) and (2) correspond to the lines $z=0.05 \mathrm{~m} / \mathrm{s}$ and $z=0 \mathrm{~nm}$ respectively; the conservative part (3) corresponds to the line $z=0 \mathrm{~m} / \mathrm{s}$.

ters $g_{i j}^{(1)}$ and $g_{i j}^{(2)}$. However, this approach is not suitable because two completely different sets of coefficients might define very similar functions on the interval of the actual engaged tip motion, $\left[A^{\mathrm{min}, \mathrm{e}} ; A^{\mathrm{max}, \mathrm{e}}\right]$, where $A^{\mathrm{max}}=\max A(t)=$ $\max z(t)$. As numerical simulations have shown, the error function does not have a well-defined global minimum and it is highly sensitive to reconstruction errors. An alternative approach is to minimize a mean square error function in real space

$$
\int_{A^{\mathrm{min}, \mathrm{e}}\left(\alpha_{2}\right)}^{A^{\max , \mathrm{e}}\left(\alpha_{2}\right)}\left[\tilde{F}_{1}\left(z^{\mathrm{e}}\left(\alpha_{2}\right)\right)-\tilde{F}_{2}\left(z^{\mathrm{e}}\left(\alpha_{2}\right) ; k_{2}\right)\right]^{2} d z,
$$

which in most regimes of the tip motion has only one global minimum lying in the deep valley defined by the curve $\alpha_{2}^{\text {true }} k_{2}^{\text {true }}$. Moreover, increasing the reconstructed polynomial power, $P_{z}$, makes this valley deeper and hence more resistant to noise. This method allows for the estimation of the product $\alpha_{2} k_{2}$ with higher accuracy than $\alpha_{2}$ and $k_{2}$ separately.

Figure 3 shows the absolute value of the relative error

$$
\eta=1-k_{2} \alpha_{2} / k_{2}^{\text {true }} \alpha_{2}^{\text {true }}
$$

plotted in the plane of maximum free oscillation energy $E^{\text {max }, \mathrm{f}}=\left(k_{1}\left(A_{1}^{\max , \mathrm{f}}\right)^{2}+k_{2}\left(A_{2}^{\max , \mathrm{f}}\right)^{2}\right) / 2$ and the ratio $R=$ $h / A^{\text {max,f }}$. The relative calibration error is small over a wide range of oscillation energy and probe height for both soft
(Figure 3a) and stiff (Figure 3b) cantilevers. The regions of lower error correspond to a large value of the ratio $A_{1}^{\max , \mathrm{f}} / A_{2}^{\max , \mathrm{f}}$ (Figure 3c and Figure 3d). Experimentally, one can check the stability of calibration by comparing different probe heights and oscillation energies. Finally, the stiff cantilever has a wider region of low error because a higher oscillation energy effectively weakens the nonlinearity.

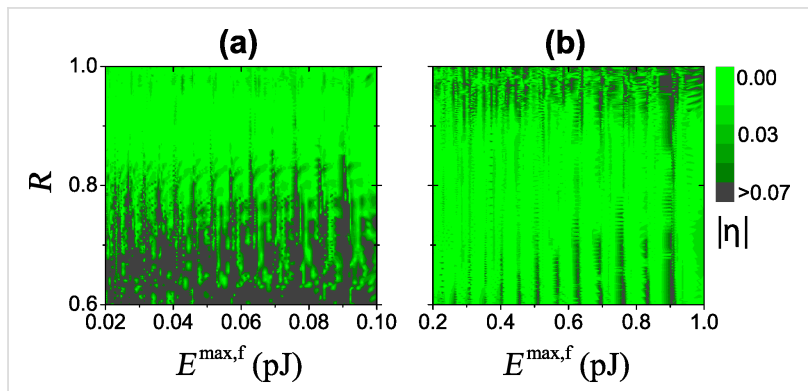

(c)

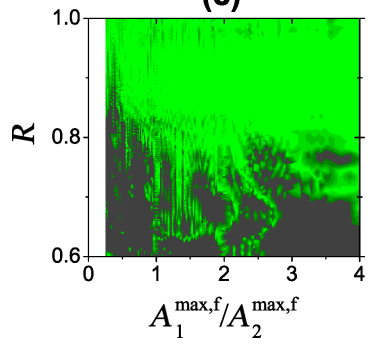

(d)

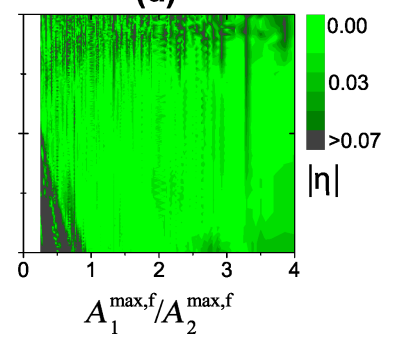

Figure 3: Absolute value of the relative calibration error $\eta$ of $k_{2} \alpha_{2}$ as a function of the ratio $R=h / A^{\text {max,f }}$, total maximum free oscillation energy $E^{\text {max,f }}$ (top row) and the ratio of maximum free amplitudes $A_{1}^{\max , \mathrm{f}} / A_{2}^{\max , \mathrm{f}}$ (bottom row) for the soft (a), (c) and stiff (b), (d) cantilever, respectively.

\section{Calibration by using a linear tip-surface force}

When the interval of the engaged tip motion is small, the tip-surface force (Equation 5) can be linearized. In this case, it is possible to obtain the explicit expression for the stiffness by using a linear model $\tilde{F}$ with one unknown parameter $g_{10}$

$$
k_{2}=\left|\frac{\hat{G}_{1}(\omega)}{\hat{G}_{2}(\omega)}\right|\left|\frac{\Delta \hat{V}_{1}(\omega)}{\Delta \hat{V}_{2}(\omega)}\right| k_{1} .
$$

If $g_{01}$ is used instead, $k_{2}$ should be additionally multiplied by $\omega_{1} / \omega_{2}$. As previously mentioned, the multimodal drive at the resonant frequencies $\omega_{1}$ and $\omega_{2}$ (more precisely, their discrete approximations defined by $\delta \omega$ ) produces enough response components to find $k_{2}$. The corresponding domain of the engaged tip motion and eigenmode sensitivity to the force are defined by the energy scale factor $k_{n}^{-1} \hat{G}_{n}\left(\omega_{n}\right)$. Therefore, calibration of the softer cantilever can be performed with higher accuracy, while for the stiff cantilever, small drive amplitudes are required for acceptable calibration results (Figure 4). Near 
the surface, the force is highly nonlinear, making the tip prone to sudden jumps to the contact. From an experimental point of view, probing only the attractive part of the interaction with small oscillation amplitudes protects the tip from possible damage since the dissipation is almost zero in this regime.

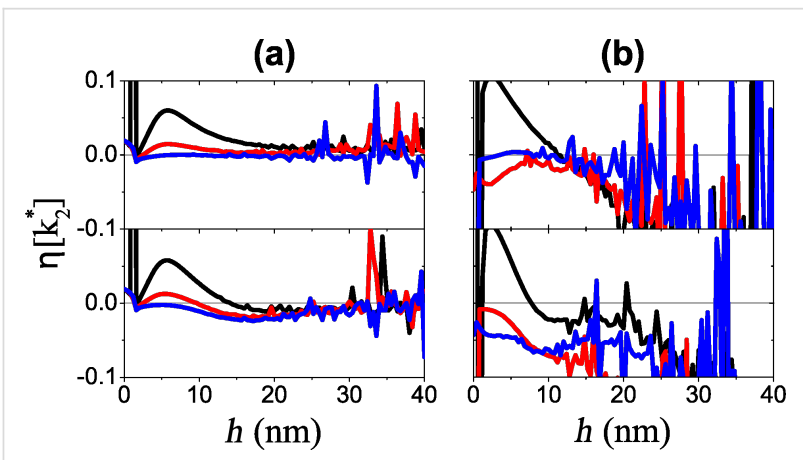

Figure 4: Relative calibration error of the calibrated stiffness $k_{2}$, $\eta=1-k_{2} / k_{2}^{\text {true }}$, using Equation 9 for two different cantilevers: (a) soft and (b) stiff, with different free eigenmode oscillations amplitudes: $A_{1}^{\mathrm{f}}=1 \mathrm{~nm}$ (top), $A_{1}^{\mathrm{f}}=3 \mathrm{~nm}$ (bottom), $A_{2}^{\mathrm{f}}=0.1 \mathrm{~nm}$ (blue), $1.0 \mathrm{~nm}$ (red) and $2.0 \mathrm{~nm}$ (black).

Finally, the linear method is dependent on the unknown higher eigenmode free amplitude, $A_{n}^{\mathrm{f}}$, which must be small for the linear approximation to be valid. Since $A_{n}^{\mathrm{f}}$ is not known a priori, one can use the following formula to try to make a rough guess given the known free amplitude of the first mode for the particular drive voltage amplitude

$$
A_{n}^{\mathrm{f}}=\left|\frac{\hat{G}^{\text {piezo }}\left(\omega_{n}\right)}{\hat{G}^{\text {piezo }}\left(\omega_{1}\right)}\right| \frac{k_{1}}{k n} \frac{Q_{n}}{Q_{1}} A_{1}^{\mathrm{f}},
$$

where $\hat{G}^{\text {piezo }}$ is a transfer function of the piezoelectric shaker.

\section{Implementation}

Summarizing the ideas presented, an experimental implementation of the proposed calibration would consist of the following steps, with related sources of possible calibration error:

1. Construct a multimode drive and measure the free motion spectrum of a cantilever, $V^{f}$. Since the free motion components can be used instead of the drive force, the real physical amplitude of the cantilever and the transfer function of the piezo shaker do not contribute to the accuracy of the method. However, as numerical calculations have shown, the method is sensitive to SNR of the measurement, performing poorly when the SNR is too small. Therefore, a drive with approximately the same SNR for all eigenmodes would be a good option.
2. Move the cantilever closer to the surface and measure its engaged motion spectrum, $V^{\mathrm{e}}$. In principle only one measured spectrum corresponding to a particular probe height is enough for calibration purposes. However, the use of spectra at different probe heights will improve calibration precision. The method may be applied to both soft and stiff cantilevers, but works best when nonlinearities are weak. Thus the amplitude contraction of the engaged cantilever oscillations with respect to the free motion should be about $10-20 \%$.

3. Choose a particular model for the tip-surface interaction and solve nonlinear system Equation 4 for unknown parameters using the measured difference spectrum $V^{\mathrm{e}}-V^{\mathrm{f}}$. If the exact expression for $F$ is unknown, ImAFM provides enough information to reconstruct it in a generic form, e.g., as power series (Equation 5). As numerical simulations have shown, a more realistic model gives better calibration with the same error function (Equation 7). Making use of any additional prior information about the cantilever also improves the accuracy of the calibration. For instance, $\omega_{n}, Q_{n}$ and $\alpha_{n}^{2} k_{n}$ can be estimated by using the thermal calibration method $[14,17]$ and the equipartition theorem (Equation 3).

Theoretically, the method should work in liquid or highdamping environments, however, experimental implementation in liquid will suffer from actuation-related effects, squeeze-film damping close to the surface and spurious resonances. [37].

\section{Conclusion}

We outlined a theoretical framework for experimental calibration of cantilever parameters by using the tip-surface force with one-point measurement and a multimodal drive. The proposed approach does not require any knowledge of the geometry of the cantilever or the form of the tip-surface interaction. The method possesses a high calibration accuracy independent of the a priori unknown amplitude of the higher eigenmode.

\section{Acknowledgements}

This work is supported by KTH, Nordita, DOE, VR VCB 6212012-2983 and the Knut and Allice Wallenberg Foundation.

\section{References}

1. Binnig, G.; Quate, C. F.; Gerber, C. Phys. Rev. Lett. 1986, 56, 930-933. doi:10.1103/PhysRevLett.56.930

2. Burnham, N. A.; Colton, R. J.; Pollock, H. M. Nanotechnology 1993, 4, 64. doi:10.1088/0957-4484/4/2/002

3. Butt, H.-J.; Cappella, B.; Kappl, M. Surf. Sci. Rep. 2005, 59, 1-152. doi:10.1016/j.surfrep.2005.08.003

4. Stark, R. W.; Heckl, W. M. Surf. Sci. 2000, 457, 219-228. doi:10.1016/S0039-6028(00)00378-2 
5. Gigler, A. M.; Dietz, C.; Baumann, M.; Martinez, N. F.; García, R.; Stark, R. W. Beilstein J. Nanotechnol. 2012, 3, 456-463. doi:10.3762/bjnano.3.52

6. Proksch, R. Appl. Phys. Lett. 2006, 89, 113121. doi:10.1063/1.2345593

7. Martinez, N. F.; Patil, S.; Lozano, J. R.; García, R. Appl. Phys. Lett. 2006, 89, 153115. doi:10.1063/1.2360894

8. Rupp, D.; Rabe, U.; Hirsekorn, S.; Arnold, W. J. Phys. D: Appl. Phys. 2007, 40, 7136-7145. doi:10.1088/0022-3727/40/22/041

9. Lozano, J. R.; García, R. Phys. Rev. Lett. 2008, 100, 076102. doi:10.1103/PhysRevLett.100.076102

10. Aksoy, M. D.; Atalar, A. Phys. Rev. B 2011, 83, 075416. doi:10.1103/PhysRevB.83.075416

11. Martinez-Martin, D.; Herruzo, E. T.; Dietz, C.; Gomez-Herrero, J.; García, R. Phys. Rev. Lett. 2011, 106, 198101. doi:10.1103/PhysRevLett.106.198101

12. Meyer, G.; Amer, N. M. Appl. Phys. Lett. 1990, 57, 2089-2091. doi:10.1063/1.103950

13. Cleveland, J. P.; Manne, S.; Bocek, D.; Hansma, P. K. Rev. Sci. Instrum. 1993, 64, 403-405. doi:10.1063/1.1144209

14. Hutter, J. L.; Bechhoefer, J. Rev. Sci. Instrum. 1993, 64, 1868-1873. doi:10.1063/1.1143970

15. Sader, J. E.; Larson, I.; Mulvaney, P.; White, L. R. Rev. Sci. Instrum. 1995, 66, 3789-3798. doi:10.1063/1.1145439

16. Higgins, M. J.; Proksch, R.; Sader, J. E.; Polcik, M.; Ms Endoo, S.; Cleveland, J. P.; Jarvis, S. P. Rev. Sci. Instrum. 2006, 77, 013701. doi:10.1063/1.2162455

17. Butt, H.-J.; Jaschke, M. Nanotechnology 1995, 6, 1. doi:10.1088/0957-4484/6/1/001

18. Sader, J. E.; Chon, J. W. M.; Mulvaney, P. Rev. Sci. Instrum. 1999, 70, 3967-3969. doi:10.1063/1.1150021

19. Stark, R. W. Rev. Sci. Instrum. 2004, 75, 5053-5055. doi:10.1063/1.1808058

20. Schäffer, T. E. Nanotechnology 2005, 16, 664. doi:10.1088/0957-4484/16/6/007

21. Liu, Y.; Guo, Q.; Nie, H.-Y.; Lau, W. M.; Yang, J. J. Appl. Phys. 2009, 106, 124507. doi:10.1063/1.3269703

22. Parkin, J. D.; Hähner, G. Nanotechnology 2013, 24, 065704. doi:10.1088/0957-4484/24/6/065704

23. Kiracofe, D.; Raman, A. J. Appl. Phys. 2010, 107, 033506. doi:10.1063/1.3284206

24. Allen, M. S.; Sumali, H.; Penegor, P. C. J. Dyn. Syst., Meas., Control 2009, 131, 064501. doi:10.1115/1.4000160

25. Burnham, N. A.; Chen, X.; Hodges, C. S.; Matei, G. A.; Thoreson, E. J.; Roberts, C. J.; Davies, M. C.; Tendler, S. J. B. Nanotechnology 2003, 14, 1. doi:10.1088/0957-4484/14/1/301

26. Lozano, J. R.; Kiracofe, D.; Melcher, J.; Garcia, R.; Raman, A. Nanotechnology 2010, 21, 465502. doi:10.1088/0957-4484/21/46/465502

27. Platz, D.; Tholén, E. A.; Pesen, D.; Haviland, D. B. Appl. Phys. Lett. 2008, 92, 153106. doi:10.1063/1.2909569

28. Borysov, S. S.; Platz, D.; de Wijn, A. S.; Forchheimer, D.; Tolén, E. A.; Balatsky, A. V.; Haviland, D. B. Phys. Rev. B 2013, 88, 115405. doi:10.1103/PhysRevB.88.115405

29. Sugimoto, Y.; Innami, S.; Abe, M.; Custance, O.; Morita, S. Appl. Phys. Lett. 2007, 91, 093120. doi:10.1063/1.2775806

30. Mokaberi, B.; Requicha, A. A. G. IEEE Trans. Autom. Sci. Eng. 2006, 3, 199-207. doi:10.1109/TASE.2006.875534

31. Hutter, C.; Platz, D.; Tholén, E. A.; Hansson, T. H.; Haviland, D. B.

Phys. Rev. Lett. 2010, 104, 050801.

doi:10.1103/PhysRevLett.104.050801
32. Forchheimer, D.; Platz, D.; Tholén, E. A.; Haviland, D. B. Phys. Rev. B 2012, 85, 195449. doi:10.1103/PhysRevB.85.195449

33. Platz, D.; Forchheimer, D.; Tholén, E. A.; Haviland, D. B. Beilstein J. Nanotechnol. 2013, 4, 352-360. doi:10.3762/bjnano.4.41

34. Hindmarsh, A. C.; Brown, P. N.; Grant, K. E.; Lee, S. L.; Serban, R.; Shumaker, D. E.; Woodward, C. S. ACM Trans. Math. Software 2005, 31, 363-396. doi:10.1145/1089014.1089020

35. Derjaguin, B. V.; Muller, V. M.; Toporov, Yu. P. J. Colloid Interface Sci. 1975, 53, 314-326. doi:10.1016/0021-9797(75)90018-1

36. Gotsmann, B.; Seidel, C.; Anczykowski, B.; Fuchs, H. Phys. Rev. B 1999, 60, 11051-11061. doi:10.1103/PhysRevB.60.11051

37. Baró, A. M.; Reifenberger, R. G. Atomic Force Microscopy in Liquid: Biological Applications; Wiley, 2012. doi:10.1002/9783527649808

\section{License and Terms}

This is an Open Access article under the terms of the Creative Commons Attribution License

(http://creativecommons.org/licenses/by/2.0), which permits unrestricted use, distribution, and reproduction in any medium, provided the original work is properly cited.

The license is subject to the Beilstein Journal of

Nanotechnology terms and conditions:

(http://www.beilstein-journals.org/bjnano)

The definitive version of this article is the electronic one which can be found at: doi:10.3762/bjnano.5.200 